\title{
Age-dependent Homeostatic Plasticity of GABAergic Signaling in Developing Retinal Networks
}

\author{
Matthias H. Hennig, ${ }^{1}$ John Grady, ${ }^{2}$ James van Coppenhagen, ${ }^{2}$ and Evelyne Sernagor ${ }^{2}$ \\ ${ }^{1}$ Institute for Adaptive and Neural Computation, School of Informatics, University of Edinburgh, Edinburgh EH8 9AB, United Kingdom, and ${ }^{2}$ Institute of \\ Neuroscience, Medical School, Newcastle University, Newcastle upon Tyne NE2 4HH, United Kingdom
}

Developing retinal ganglion cells fire in correlated spontaneous bursts, resulting in propagating waves with robust spatiotemporal features preserved across development and species. Here we investigate the effects of homeostatic adaptation on the circuits controlling retinal waves. Mouse retinal waves were recorded in vitro for up to $35 \mathrm{~h}$ with a multielectrode array in presence of the $\mathrm{GABA}_{\mathrm{A}}$ antagonist bicuculline, allowing us to obtain a precise, time-resolved characterization of homeostatic effects in this preparation. Experiments were performed at $\mathrm{P} 4-\mathrm{P} 6$, when $\mathrm{GABA}_{\mathrm{A}}$ signaling is depolarizing in ganglion cells, and at $\mathrm{P} 7-\mathrm{P} 10$, when $\mathrm{GABA}_{\mathrm{A}}$ signaling is hyperpolarizing. At all ages, bicuculline initially increased the wave sizes and other activity metrics. At P5-P6, wave sizes decreased toward control levels within a few hours while firing remained strong, but this ability to compensate disappeared entirely from P7 onwards. This demonstrates that homeostatic control of spontaneous retinal activity maintains specific network dynamic properties in an age-dependent manner, and suggests that the underlying mechanism is linked to $\mathrm{GABA}_{\mathrm{A}}$ signaling.

\section{Introduction}

Developing CNS networks exhibit patterned spontaneous bursting activity that is remarkably similar across systems (Blankenship and Feller, 2010). In the developing retina, RGCs fire in spontaneous bursts of action potentials that are correlated between neighbors, resulting in propagating waves (Meister et al., 1991). The earliest embryonic mammalian retinal waves are generated via gap junctions (Stage I), followed by Stage II, when they are driven by cholinergic amacrine cells [from late gestation until postnatal day 9 (P9) in mouse] (Torborg and Feller, 2005). Waves become subsequently driven by glutamatergic networks (Stage III), from P10 onwards in mouse. Depolarizing $\mathrm{GABA}_{\mathrm{A}}$ signaling becomes involved in controlling mouse retinal waves around $\mathrm{P} 4$, shifting to mature hyperpolarization between P7 and P9 (Zhang et al., 2006; Barkis et al., 2010). Cholinergic waves have remarkably robust spatiotemporal features preserved across both development and species (Sun et al., 2008b; Hennig et al., 2009), suggesting that they may be resilient to changes imposed by the environment. It is therefore an important question whether retinal waves are stabilized in a neural activity-dependent manner.

Received June 19, 2011; accepted July 8, 2011.

Author contributions: M.H.H., J.G., and E.S. designed research; J.G., J.V.C., and E.S. performed research; M.H.H. and J.G. contributed unpublished reagents/analytic tools; M.H.H., J.G., J.v.C., and E.S. analyzed data; M.H.H. and E.S. wrote the paper.

This work was funded by Newcastle University Faculty of Medical Sciences Graduate School (E.S., J.G., J.v.C.), Wellcome Trust Grant 090194/Z/09/Z (E.S., J.G.), Engineering and Physical Sciences Research Council, Code Analysis, Repository and Modelling for e-Neuroscience grant (EP/E002331/1) (E.S.), and Medical Research Council Grant G0900425 (M.H.H.). We thank M. O'Donovan and M. Van Rossum for their comments on the manuscript.

Correspondence should be addressed to Evelyne Sernagor, Institute of Neuroscience, Medical School, Newcastle University, Framlington Place, Newcastle upon Tyne NE2 4HH, UK. E-mail: evelyne.sernagor@ncl.ac.uk.

DOI:10.1523/JNEUROSCI.3112-11.2011

Copyright $\odot 2011$ the authors $\quad 0270-6474 / 11 / 3112159-06 \$ 15.00 / 0$
It is well known from studies using neuronal cultures that chronic changes in activity levels (including blockade of GABAergic inhibition) can induce homeostatic changes through modification of intrinsic neuronal properties and synaptic strength (Turrigiano et al., 1998; Turrigiano and Nelson, 2004). Except for the spinal cord, however (Chub and O'Donovan, 1998; Wilhelm et al., 2009), very little is known about homeostatic adaptation following activity perturbation in intact networks. The only evidence of acute homeostatic compensation in retinal networks comes from two studies. Rabbit Stage I waves can resume upon cholinergic nicotinic blockade of Stage II waves immediately after the switch from Stage I to II (Syed et al., 2004). In mouse, small patches of synchronized activity can be detected at P5 following 5-10 h of continuous cholinergic nicotinic blockade (Stacy et al., 2005).

Here we have investigated the effect of prolonged exposure to the $\mathrm{GABA}_{\mathrm{A}}$ antagonist bicuculline on retinal wave dynamics before the $\mathrm{GABA}_{\mathrm{A}}$ shift $(\mathrm{P} 4-\mathrm{P} 6)$ and once $\mathrm{GABA}_{\mathrm{A}}$ starts shifting polarity $(\mathrm{P} 7-\mathrm{P} 10)$. We report that cholinergic retinal waves are under homeostatic control, and our results indicate that $\mathrm{GABA}_{\mathrm{A}}$ signaling is implicated in this regulation.

\section{Materials and Methods}

Experimental procedures. Neonatal C57BL/6 mice were used in this study. All animal procedures were conducted under the UK Home Office, Animals (Scientific Procedures) Act 1986. Mouse pups were killed by cervical dislocation and enucleated before retinal isolation. The isolated retina was then transferred to the experimental chamber and placed, RGC layer facing down, onto MEAs consisting of 60 titanium nitride electrodes (30 $\mu \mathrm{m}$ diameter, $200 \mu \mathrm{m}$ spacing) arranged in an $8 \times 8$ grid (without corners) on indium tin oxide substrate (Multi Channel Systems). Better coupling between the tissue and the electrodes was achieved by placing a small piece of polyester membrane filter ( $5 \mu \mathrm{m}$ pores) (Sterlitech) on the retina followed by a slice anchor holder (Warner Instruments). The retina was kept at $32^{\circ} \mathrm{C}$ and continuously perfused $(0.5-1 \mathrm{ml} / \mathrm{min})$ with 
artificial CSF (aCSF) containing the following (in mM): $118 \mathrm{NaCl}, 25 \mathrm{NaHCO}_{3}, 1 \mathrm{NaH}_{2} \mathrm{PO}_{4}$, $3 \mathrm{KCl}, 1 \mathrm{MgCl}_{2}, 2 \mathrm{CaCl}_{2}$, and 10 glucose, equilibrated with $95 \% \mathrm{O}_{2}$ and $5 \% \mathrm{CO}_{2}$. Control waves were recorded after the tissue was allowed to settle for $1-2 \mathrm{~h}$ on the array to ensure that spontaneous activity had reached steady state. Bicuculline was added directly to the perfusate. The solution was not recycled, so that the tissue was constantly perfused with fresh aCSF. At the end of the first day of recordings, the MEA was disconnected from the amplifiers and the in-line heater was turned off. The retina on the MEA was kept at room temperature and perfused with fresh oxygenated aCSF (with or without bicuculline) overnight. On day 2, the MEA was reconnected to the amplifiers and the aCSF brought back up to $32^{\circ} \mathrm{C}$. Recordings were resumed after $\sim 1 \mathrm{~h}$. We recorded healthy spontaneous waves in vitro for up to $35 \mathrm{~h}$, our longest trial (including control, bicuculline, and wash).

Signals were amplified (gain 1200) and acquired using a 128-channel analog to digital converter (Multi Channel Systems MC_Card). Signals were digitized at $10 \mathrm{kHz}$ and acquired with a $200 \mathrm{~Hz}$ high-pass filter using the software MC_Rack (Multi Channel Systems). The time of occurrence of spontaneous spikes was threshold-detected with MC_Rack (the typical threshold was at signal amplitude that is three times the baseline noise). Hence, the firing rate on each electrode reflects the overall activity level generated by all RGCs on that same electrode (typically $4-5$ cells). Using the software MC_Data Tools (Multi Channel Systems), the times of spike occurrence were converted into text files for further analysis.

Spikes originating from individual RGCs were extracted on a number of channels using the Matlab-based software package Waves_clus (Quiroga et al., 2004). Time stamp files from these individual spike clusters were analyzed using a custom-written burst detection algorithm (see below).

Experiments were conducted at P4 (1 d: $n=2), \mathrm{P} 5(1 \mathrm{~d}: n=2 ; 2 \mathrm{~d}: n=$ 3), P6 (2 d: $n=2)$, P7 ( $1 \mathrm{~d}: n=1), \mathrm{P} 8(1 \mathrm{~d}: n=1)$, P9 $(2 \mathrm{~d}: n=2)$, and P10 (2 d: $n=1)$. Spike datasets are publicly available for download via the CARMEN portal (https://portal.carmen.org.uk/).

Data analysis. Retinal waves were detected and analyzed using custom software written in Matlab (Version 7.21; MathWorks). Briefly, first spike bursts were detected on each electrode separately, which were then grouped into waves based on temporal overlap (Hennig et al., 2009). Burst onset was defined by the length of the first interspike interval (ISI) and the spike count in a fixed window starting at the first potential burst spike. Specifically, all ISIs in one spike train were ranked as $R(t)$, and the probability distribution $P(C)$ of spike counts in a $1 \mathrm{~s}$ time window was obtained. Then, each spike train was analyzed spike by spike, and the start of a burst was defined as a spike followed by an ISI with rank $R(t)<\theta_{R}$, with $\theta_{R}=0.5$, and where the spike count $C(t, t+1 \mathrm{~s})>\theta_{C}$. The spike count threshold $\theta_{C}$ was set to the value where $P(C)=0.05$, and calculated individually for each channel to account for variations in mean firing rates. The end of a burst was determined as the time of the spike where $C<\theta_{C} / 2$. This method could reliably detect bursts and their durations and was insensitive to the large differences in average and peak firing rates between different electrodes.

Waves were detected as temporally overlapping groups of bursts. To prevent splitting of waves due to short bursts, minimum burst duration of $5 \mathrm{~s}$ was imposed during wave detection. Waves consisting of activity on just a single electrode were not considered in the analysis of wave sizes, but were included in all other metrics. Wave speed was measured for each wave by recording the relative latency of each burst that occurred in the neighboring eight electrodes of each bursting electrode and dividing these latencies by the corresponding distance on the array. The median values presented in this paper were obtained from the pooled distribution of all pairs and waves in a particular interval of the recording. All statistical comparisons were performed using the two-sample Kolmogorov-Smirnov (KS) test.

\section{Results}

First, we examined whether retinal waves remain stable over the course of several hours in vitro (Fig. 1). Control experiments at P5, P6, and P7 showed that spatial wave properties were essentially unchanged during $>6 \mathrm{~h}$ of continuous recording, as the distribution of wave sizes did not change significantly during this period ( $p \geq 0.05$, two-sample KS test comparing the first and second halves of the recording). At the same time, there is a gradual decrease of the interburst interval (IBI), of interwave intervals (IWIs), and of the wave propagation velocity $(p<$ $0.005, \mathrm{KS}$ test).

On day 2, wave sizes, IWIs, IBIs, and wave speeds are stable at a lower level than at the start of the experiment $(p<0.005, \mathrm{KS}$ test). Median burst durations are similar throughout the $2 \mathrm{~d}$ experiments, with distributions not changing during day 1 , but becoming broader on day 2 ( $p<0.005$, KS test). This shows that neural activity is not entirely stable in these prolonged in vitro recordings from excised retinas and, especially on day 2 , shows signs of increased network excitability perhaps due to cell depolarization and/or tissue deterioration. At the same time, important wave properties are preserved for many hours, establishing a baseline for interpretation of the homeostasis experiments described in the next section.

To examine whether the developing retinal network has the capacity to compensate for prolonged activity changes, we recorded retinal waves during sustained exposure to the $\mathrm{GABA}_{\mathrm{A}}$ 

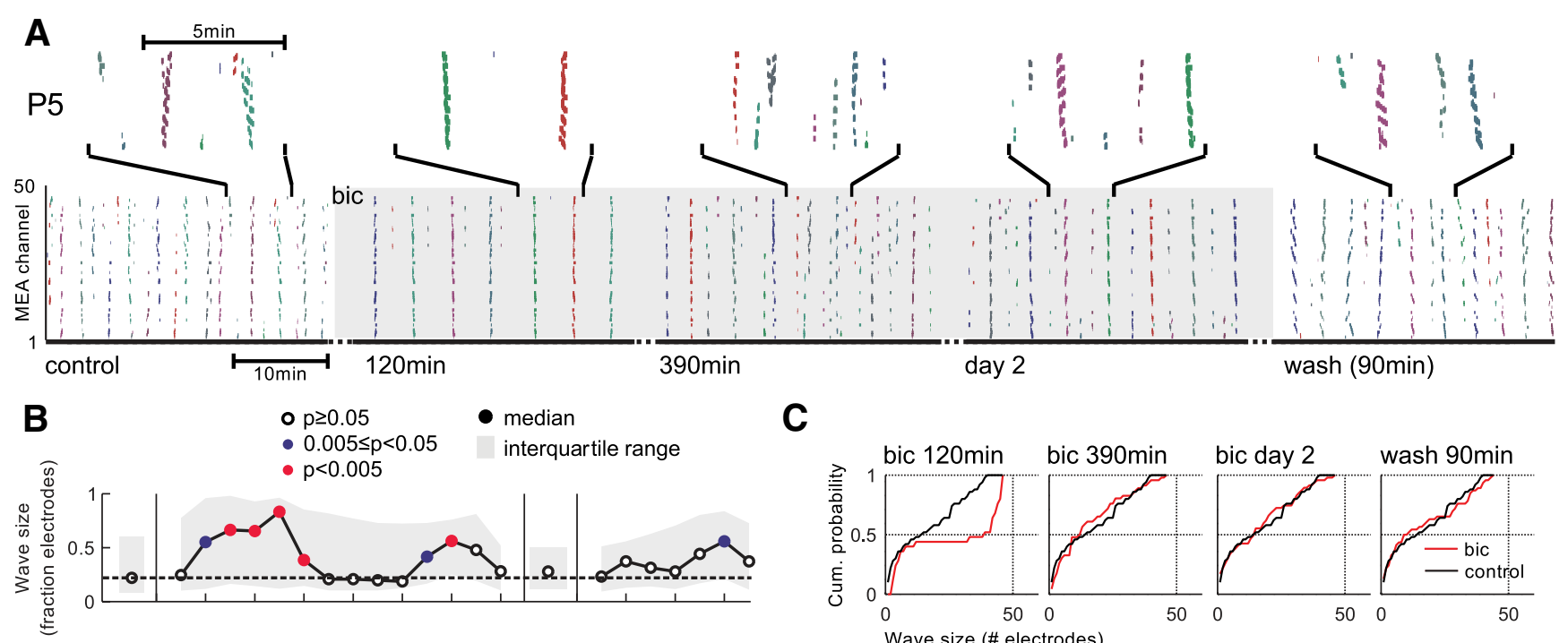

C
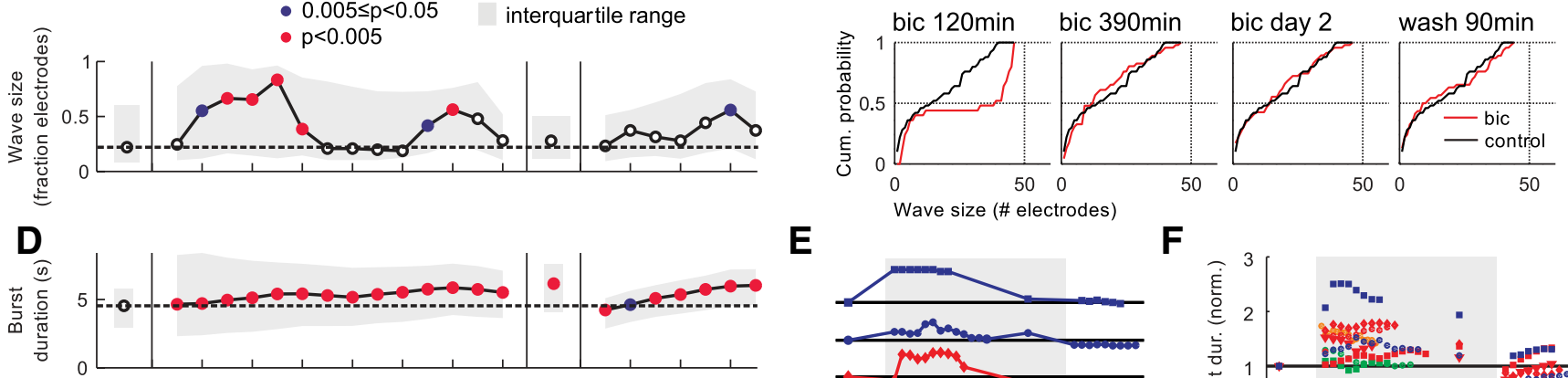

E
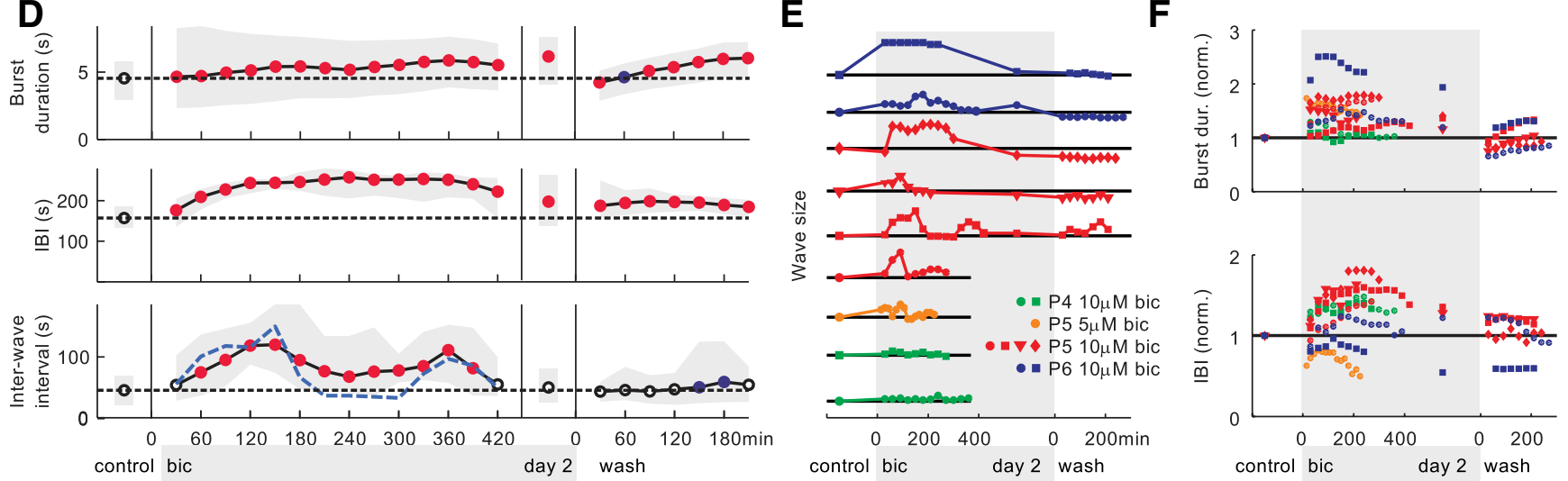

Figure 2. Prolonged bicuculline exposure induces homeostatic compensation of retinal wave sizes at P5/P6.A, Raster plots of bursts at different time points before, during (shaded area), and after application of $10 \mu \mathrm{m}$ bicuculline at P5. B, D, Medians (circles) and interquartile ranges (shaded areas) of wave sizes $(\boldsymbol{B})$ and burst duration, interburst interval, and interwave interval (D) before, during, and after bicuculline application for the dataset shown in $A$. Data points represent $1 \mathrm{~h}$ segments in steps of $30 \mathrm{~min}$, and the data for control and day 2 bicuculline was pooled (at least $2 \mathrm{~h}$ recording time). The color of each data point indicates the $p$ value of a two-sample Kolmogorov-Smirnov test comparing the underlying distribution with the distribution during control (leftmost data point). Empty circles indicate no significant difference. The dotted blue line in the interwave interval plot in $\boldsymbol{D}$ (bottom graph) is the scaled median wave size to illustrate the correlation of these metrics. C, Cumulative wave size distributions for $1 \mathrm{~h}$ segments for the same dataset (black: control; red: bicuculline). $\boldsymbol{E}$, Median wave sizes in nine experiments at P4, P5, and P6. F, Normalized median burst durations and IBIs for the experiments in $\boldsymbol{E}$.

blocker bicuculline $(10 \mu \mathrm{M})$. At P5, bicuculline had an immediate prominent positive effect on wave sizes, with disappearance of medium-size waves and prevalence of large ones within the first hour of exposure, suggesting that GABA dampens propagation across the network through shunting inhibition (Fig. 2A-C) (Sernagor et al., 2003; Syed et al., 2004). The full effect of bicuculline is delayed by $\sim 30 \mathrm{~min}$, which we attribute to the slow solution exchange in the recording chamber (the first "bicuculline" recording arbitrarily starts when the perfusion tube is switched from control aCSF to bicuculline) and also to the slow reorganization of the network dynamics. Unperturbed waves are frequent and highly variable in sizes and trajectories, such that the slow refractory mechanism controlling waves at this age initially prevents rapid network synchronization in bicuculline. Bicuculline also caused a rapid $\sim 1.5$-fold increase in wave propagation speed (not illustrated), supporting the idea that GABA has a role in controlling activity propagation.

The increase of wave sizes was not permanent during continued exposure to bicuculline, but, within hours, a clear compensatory effect became visible (Fig. $2 A-C$ ). Median wave sizes decreased from initially high values back toward the lower con- trol values, and the wave size distributions eventually became statistically indistinguishable from control ( $p \geq 0.05$, KS test; Fig. $2 B, C$ ). This compensation remained stable for as long as recordings lasted. Upon drug washout, wave sizes returned to control values. In the example shown in Figure $2 A-C$, the compensatory effect showed slow oscillations, suggesting the presence of feedback control, but generally the time course of compensation and oscillations was not consistent across preparations (Fig. 2E).

Compensation of wave sizes was observed in all seven experiments we conducted at P5 or P6 (Fig. 2E), but with a highly variable time course between preparations. Onset of compensation ranged from just $2 \mathrm{~h}$ at $\mathrm{P} 5$ to a whole day at P6. The reasons for this variability could not be assessed due to uncertainties regarding the precise birth date of the animals and the rapid developmental changes in network structure and function at these ages. At P4, the effect of bicuculline on wave sizes was too weak to allow for systematic comparison, although bicuculline clearly increased IBIs, indicating the presence of functional $\mathrm{GABA}_{\mathrm{A}}$ synapses at this age.

At the same time, bicuculline application causes a gradual increase of IBIs (Fig. 2D,F), indicating a slow concomitant re- 


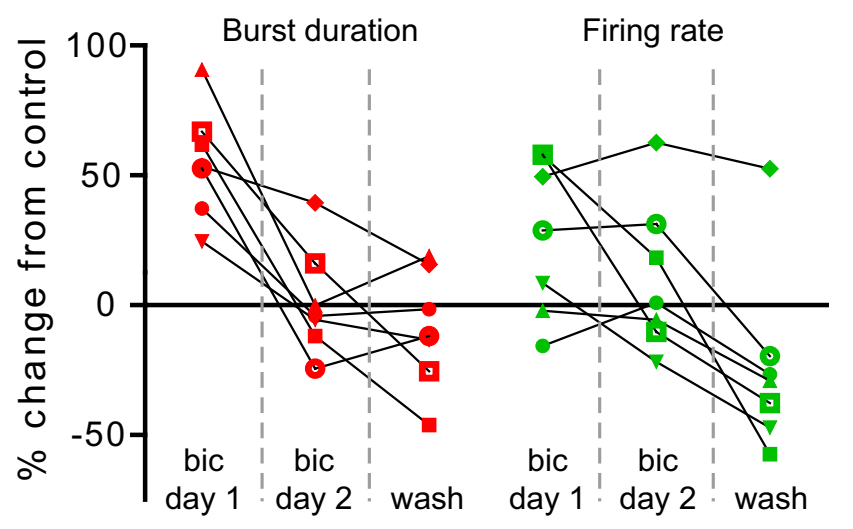

Figure 3. Bursting behavior in individual RGCs undergo homeostatic compensation as well. The figure illustrates the changes from control for burst duration (red symbols) and firing rate (green symbols) for seven individual RGCs (each one represented by a different symbol) in a P5 retina 90 min after the addition of bicuculline (bic day 1 ), on the next morning (bic day 2), and $180 \mathrm{~min}$ after the beginning of bicuculline washout (wash). Each dataset consists of $30 \mathrm{~min}$ of continuous recording.

duction of network excitability. Burst duration distributions initially showed an additional peak toward higher values, returning within a few hours to lower values with unimodal shape, but not to control levels (Fig. 2D,F). Interestingly, as soon as bicuculline was washed out, burst durations became significantly shorter, slowly rising back to a steady baseline level within $2-3 \mathrm{~h}$, perhaps revealing the presence of a compensatory mechanism at work after prolonged blockade of $\mathrm{GABA}_{\mathrm{A}}$ receptors. As for wave sizes, there was substantial variability of the magnitude of these effects between preparations (Fig. $2 F$ ). An exception was an experiment with $5 \mu \mathrm{M}$ bicuculline at P5, where wave sizes were compensated but IBIs decreased below control in bicuculline, perhaps suggesting a dose-dependent effect. IBIs decreased in one experiment at P6 as well. The latter behavior is typical for older retinas (see below), suggesting this retina may have been more mature. Mean firing rates also increased in bicuculline, and showed a slight reduction after several hours, but not back to the control level. Wave propagation speed remained elevated as long as bicuculline was present (not illustrated). In addition, there was a significant correlation between wave sizes and IWIs (Fig. 2D; see below).

To determine whether these compensatory changes observed at the global network level are also reflected in individual RGCs, we have performed spike sorting on a number of electrodes to assign different spike waveforms to individual RGCs. This was done in control conditions, during the initial exposure to bicuculline (before compensation occurs), during exposure to bicuculline on the second day, and during wash. Figure 3 illustrates the changes in bursting behavior in seven RGCs from a P5 retina across the different experimental conditions. Burst durations initially increased in all cells upon exposure to bicuculline (measured at 90-120 min after the start of bicuculline application), but decreased back to control levels or even lower for five cells on the second day. Upon bicuculline washout (measured 180-210 min after the wash onset), burst durations decreased further in four cells, and in the remaining cells they did either not change or slightly increased. Firing rates followed the same general pattern, but with more variability. These changes corroborate those we reported at the global network level (Fig. 2), demonstrating that homeostatic effects can also be detected at the level of individual RGCs, but with substantial variability between neurons. Hence biased sampling of RGCs may, at least in part, explain the variability observed between preparations where activity was measured by thresholding the recorded signals on individual electrodes (Fig. 2).

The ability for homeostatic compensation of the bicucullineinduced increase in wave sizes disappeared from P7 onwards. As shown in Figure $4 A-C$, at P9, bicuculline caused a strong and rapid increase in wave sizes ( $p<0.005$, KS test), with waves remaining larger and longer for as long as the drug was present. Upon bicuculline washout, wave sizes first rapidly decreased toward control levels, but then increased again and remained at an elevated level $(0.005 \leq p<0.05$, KS test). Our recordings during wash were not long enough to assess whether wave sizes eventually return toward control levels or below these, as observed in control recordings (Fig. $1 B$ ). However, waves were still significantly larger than control $5 \mathrm{~h}$ after drug washout, suggesting that long-term bicuculline exposure induces long-lasting changes at this age (Fig. $4 B$ ). Similar effects were observed in a total of five experiments conducted between P7 and P10 (Fig. 4E). At P7, some decrease of waves sizes toward the end of the experiment is still visible, suggesting that compensation may still be possible at this age to some extent. At P10, the effect of bicuculline on wave sizes was weak because waves were already large during control, but nevertheless, as for the other ages, waves were also larger for as long as bicuculline was present.

As for wave sizes, other activity parameters rapidly changed at P9 as soon as bicuculline was applied without any further change as long as the drug was present (Fig. $4 D, F$ ). Burst durations increased significantly ( $p<0.005, \mathrm{KS}$ test), and interburst intervals were reduced to about half the control value $(p<0.005, \mathrm{KS}$ test), clearly indicating that bicuculline prevents the inhibitory effect of GABA at this age (Sernagor et al., 2003; Syed et al., 2004). IWIs increased in presence of bicuculline, with some modulations over time, which were, unlike at P5, not correlated with changes in wave sizes. Wave propagation speed showed a sustained increase by about a factor of two at P7-P9, but decreased slightly at P10. Overall, none of the parameters assessed showed any sign of compensation in presence of bicuculline, but there was considerable variability in the strength of the drug effect.

Upon drug washout, burst durations returned close to control values at P9 (Fig. $4 F$ ), but their distribution was narrower than in control ( $p<0.005$, KS test), suggesting reduced variability in neural excitability following prolonged bicuculline exposure. At P10, burst durations remained elevated. Interburst intervals remained lower during wash at P9, at a level similar to that observed in control recordings (Fig. 1 B). At P10, however, they were markedly increased. Hence, while there is little indication that prolonged bicuculline exposure induces compensatory effects as observed at P5/6, the behavior during wash raises the possibility that it may cause long-lasting alterations in cellular excitability and/or synaptic efficacies.

It is noteworthy that all neural activity metrics during bicuculline exposure at P5/6 indicate a slow tendency toward decreasing neural activity, which is the opposite trend as observed under control conditions (Fig. 1). However, the time course of these changes was not directly correlated with the fluctuations in wave sizes, indicating the presence of multiple, synergistic processes controlling this homeostatic compensation process. Correlations were only observed between wave sizes and IWIs (Pearson's $r=$ $0.62-0.86, p<0.0002-0.0364, n=7$; see blue line in Fig. $2 D$, bottom trace). This correlation is not unexpected, because at this age, wave initiation and propagation is limited by a long-lasting refractory mechanism (Zheng et al., 2006). Larger waves drive more neurons into the refractory state than small waves, which in turn delay the initiation of subsequent waves in the recorded area 


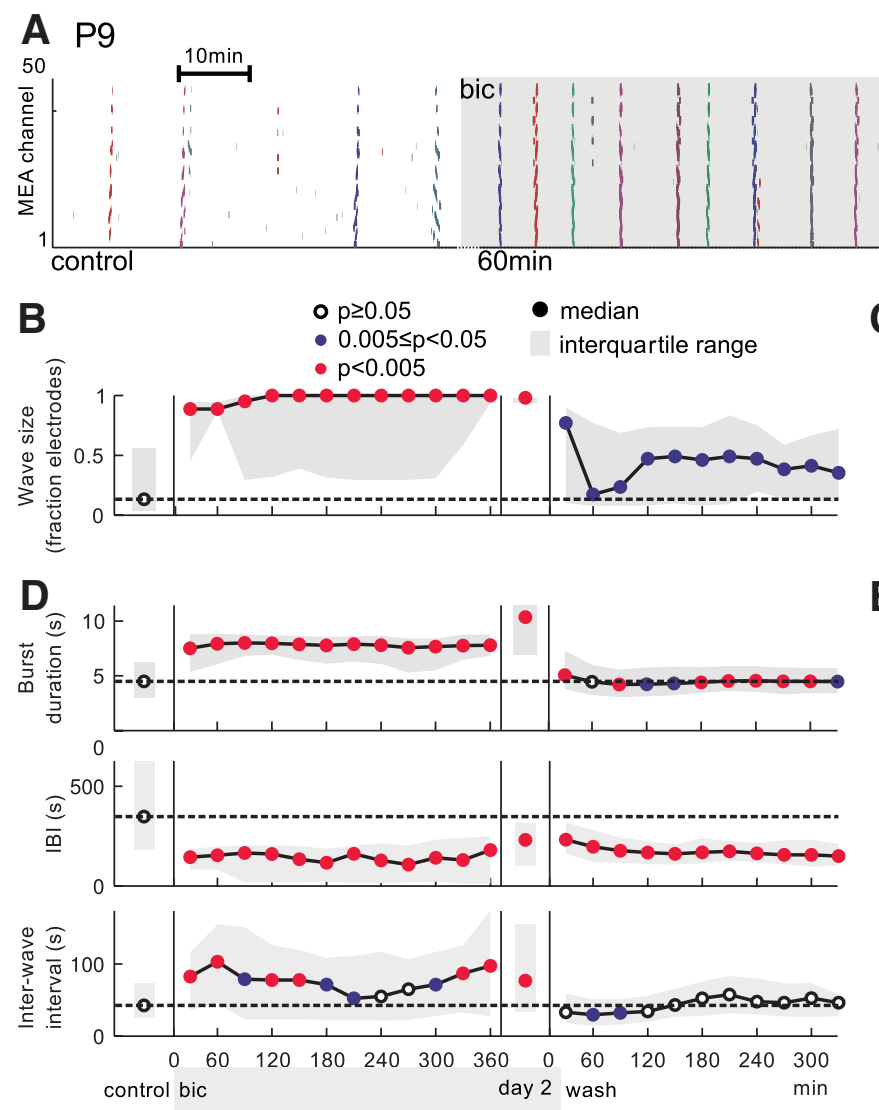

Figure 4. No compensation during prolonged bicuculline exposure at P9. $\boldsymbol{A}$, Raster plots of bursts at different time points before, during (shaded area), and after application of $10 \mu \mathrm{m}$ bicuculline. $\boldsymbol{B}, \boldsymbol{D}$, Medians (circles) and interquartile ranges (shaded areas) of wave sizes $(\boldsymbol{B})$ and burst durations, interburst intervals, and interwave intervals $(\boldsymbol{D})$ before, during, and after bicuculline application. Data are presented as in Figure 2. C, Cumulative wave size distributions for $1 \mathrm{~h}$ segments (black: control; red: bicuculline). $\boldsymbol{E}$, Median wave sizes in five experiments at P7, P8, P9, and P10. $\boldsymbol{F}$, Normalized median burst durations and IBIs for the experiments in $\boldsymbol{E}$.

(Hennig et al., 2009). Homeostatic regulation of the conductance underlying the refractory mechanism, therefore, is unlikely to contribute to wave size compensation, since this would result in a systematic change of the relationship between wave sizes and IWIs.

\section{Discussion}

This is, to our knowledge, the first detailed time-resolved characterization of the effects of homeostatic plasticity in an intact central circuit. These compensatory mechanisms are linked to $\mathrm{GABA}_{\mathrm{A}}$ signaling and disappear with the maturation of GABAergic inhibition. Importantly, our results show that the homeostatic set point is defined by a global network property (wave size distributions) rather than by local, intrinsic activity measures (average firing rate). No single change in neural activity correlates with wave size compensation, and not all aspects of the waves respond in the same manner to bicuculline, suggesting that several mechanisms act in synergy.

One possibility is that the compensation at P5-P6 may stem from changes in cation- $\mathrm{Cl}^{-}$cotransporter functionality (Blaesse et al., 2009). Supporting this hypothesis, premature KCC2 upregulation has recently been reported in the neonatal hippocampus following seizure induction (Khirug et al., 2010), and changes in $E_{\mathrm{GABA}}$ appear to mediate homeostatic synaptic plasticity in the developing spinal cord (Gonzalez-Islas et al., 2010). The intriguing abrupt disappearance of ability for homeostatic compensation around P7 coincides with the switch of $\mathrm{GABA}_{\mathrm{A}}$ response polarity in RGC somata (Zhang et al., 2006), suggesting that both processes may be triggered by a common mechanism linked to the disappearance of GABAergic signaling plasticity in maturing networks, e.g., through downregulation of an early $\mathrm{Cl}^{-}$accumulator (Zhang et al., 2007) or the possible shift from low- to highthreshold $\mathrm{Ca}^{2+}$ channels (Zhang et al., 2006).

Previous studies using genetic manipulations have shown that Stage I waves can reappear when Stage II cholinergic waves are disrupted (Stacy et al., 2005; Sun et al., 2008a) and that Stage III glutamatergic waves can emerge earlier than normal in the absence of Stage II waves (Bansal et al., 2000). Abolishing Stage III waves also induces the reappearance of Stage II-like waves (Wang et al., 2007). Here we show for the first time that homeostasis can maintain activity properties specific to particular developmental stages, and that this phenomenon can be triggered within just a few hours. This may be relevant during activity-dependent refinement of retinal projections to their targets, which depends on cholinergic retinal waves and may have an instructive role (Torborg and Feller, 2005). Homeostatic compensation can ensure the maintenance of the required dynamic properties, conferring robustness to developmental and environmental influences.

\section{References}

Bansal A, Singer JH, Hwang BJ, Xu W, Beaudet A, Feller MB (2000) Mice lacking specific nicotinic acetylcholine receptor subunits exhibit dramatically altered spontaneous activity patterns and reveal a limited role for retinal waves in forming on and off circuits in the inner retina. J Neurosci 20:7672-7681.

Barkis WB, Ford KJ, Feller MB (2010) Non-cell-autonomous factor induces 
the transition from excitatory to inhibitory GABA signaling in retina independent of activity. Proc Natl Acad Sci U S A 107:22302-22307.

Blaesse P, Airaksinen MS, Rivera C, Kaila K (2009) Cation-chloride cotransporters and neuronal function. Neuron 61:820-838.

Blankenship AG, Feller MB (2010) Mechanisms underlying spontaneous patterned activity in developing neural circuits. Nat Rev Neurosci 11:18-29.

Chub N, O’Donovan MJ (1998) Blockade and recovery of spontaneous rhythmic activity after application of neurotransmitter antagonists to spinal networks of the chick embryo. J Neurosci 18:294-306.

Gonzalez-Islas C, Chub N, Garcia-Bereguiain MA, Wenner P (2010) Gabaergic synaptic scaling in embryonic motoneurons is mediated by a shift in the chloride reversal potential. J Neurosci 30:13016-13020.

Hennig MH, Adams C, Willshaw D, Sernagor E (2009) Early-stage waves in the retinal network emerge close to a critical state transition between local and global functional connectivity. J Neurosci 29:1077-1086.

Khirug S, Ahmad F, Puskarjov M, Afzalov R, Kaila K, Blaesse P (2010) A single seizure episode leads to rapid functional activation of kcc2 in the neonatal rat hippocampus. J Neurosci 30:12028-12035.

Meister M, Wong RO, Baylor DA, Shatz CJ (1991) Synchronous bursts of action potentials in ganglion cells of the developing mammalian retina. Science 252:939-943.

Quiroga RQ, Nadasdy Z, Ben-Shaul Y (2004) Unsupervised spike detection and sorting with wavelets and superparamagnetic clustering. Neural Comput 16:1661-1687.

Sernagor E, Young C, Eglen SJ (2003) Developmental modulation of retinal wave dynamics: shedding light on the GABA saga. J Neurosci 23:7621-7629.

Stacy RC, Demas J, Burgess RW, Sanes JR, Wong RO (2005) Disruption and recovery of patterned retinal activity in the absence of acetylcholine. J Neurosci 25:9347-9357.

Sun C, Warland DK, Ballesteros JM, van der List D, Chalupa LM (2008a)
Retinal waves in mice lacking the beta 2 subunit of the nicotinic acetylcholine receptor. Proc Natl Acad Sci U S A 105:13638-13643.

Sun C, Speer CM, Wang GY, Chapman B, Chalupa LM (2008b) Epibatidine application in vitro blocks retinal waves without silencing all retinal ganglion cell action potentials in developing retina of the mouse and ferret. J Neurophysiol 100:3253-3263.

Syed MM, Lee S, Zheng J, Zhou ZJ (2004) Stage-dependent dynamics and modulation of spontaneous waves in the developing rabbit retina. J Physiol 560:533-549.

Torborg CL, Feller MB (2005) Spontaneous patterned retinal activity and the refinement of retinal projections. Prog Neurobiol 76:213-235.

Turrigiano GG, Nelson SB (2004) Homeostatic plasticity in the developing nervous system. Nat Rev Neurosci 5:97-107.

Turrigiano GG, Leslie KR, Desai NS, Rutherford LC, Nelson SB (1998) Activity-dependent scaling of quantal amplitude in neocortical neurons. Nature 391:892-896.

Wang CT, Blankenship AG, Anishchenko A, Elstrott J, Fikhman M, Nakanishi S, Feller MB (2007) GABA(A) receptor-mediated signaling alters the structure of spontaneous activity in the developing retina. J Neurosci 27:9130-9140.

Wilhelm JC, Rich MM, Wenner P (2009) Compensatory changes in cellular excitability, not synaptic scaling, contribute to homeostatic recovery of embryonic network activity. Proc Natl Acad Sci U S A 106:6760-6765.

Zhang LL, Pathak HR, Coulter DA, Freed MA, Vardi N (2006) Shift of intracellular chloride concentration in ganglion and amacrine cells of developing mouse retina. J Neurophysiol 95:2404-2416.

Zhang LL, Delpire E, Vardi N (2007) Nkccl does not accumulate chloride in developing retinal neurons. J Neurophysiol 98:266-277.

Zheng J, Lee S, Zhou ZJ (2006) A transient network of intrinsically bursting starburst cells underlies the generation of retinal waves. Nat Neurosci 9:363-371. 